\title{
Acute impact of cigarette smoking on power spectral measures of HRV
}

\author{
Mehboba Ferdous ${ }^{1}$, Sultana Ferdousi ${ }^{2}$
}

\begin{abstract}
Background: Cigarette smoking is associated with various forms of acute cardiac events such as myocardial infarction and ventricular fibrillation etc. Objective: To observe immediate impact of cigarette smoking on power spectral measures of heart rate variability (HRV) in current apparently healthy male regular cigarette smokers. Methods: This study was carried out on 120 apparently healthy male current regular cigarette smoker aged 20-55 years. To watch the intense impacts, data were recorded 5 and 30 min after completing a full stick of cigarette. Power spectral measures of HRV were recorded by a RMS digital polyrite D. Statistical analysis was done by paired sample t test. Results: LF power, LF nu \& LF/HF ratio were significantly increased and HF power \& HF nu were significantly decreased from their corresponding baseline value just 5 minutes after smoking and returned close to their baseline value after 30 minutes of smoking. Conclusion: The result of the study concludes that acceleration of sympathetic activity with simultaneous withdrawn of cardiovascular vagal adjustment happens quickly in the wake of smoking a cigarette.
\end{abstract}

Keywords: LF, HF, LF/HF, Cigarette smoking

J Bangladesh Soc Physiol. 2018, June; 13(1): 8-12 For Authors Affiliation, see end of text.

http://www.banglajol.info/index.php/JBSP DOI: http://dx.doi.org/10.3329/jbsp.v13i1.37842

\section{Introduction}

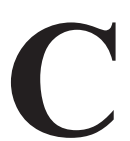

igarette smoking is a major cause of atherosclerotic disease and is one of the major risk factor for coronary heart

disease along with hypertension and lipid disorders. Among the few basic instrument centering the connection between cigarette smoking and intense cardiovascular occasions, cardiac sympathetic hyperactivity has been noted as a prevailing element in cigarette smokers. Hayano et al investigated the short and long term effects of cigarette smoking on cardiac autonomic regulation \& found decreased vagal cardiac control 3 minutes after smoking and increased sympathetic activity 10-17 minutes after smoking compared to their presmoking values ${ }^{1}$. Long term effects were also examined and compared between non-smokers and smokers. They demonstrated that smoking causes an acute and transient decrease in vagal cardiac control in smokers ${ }^{1}$.

Heart rate changeability is the most delicate and subjective marker for both sympathetic and parasympathetic action. In clinical practice, power

Received 15 Nov. 2017; $\quad$ Accepted 02 Feb. 2018 spectral analysis of HRV based on 5 minute ECG recording are widely used to assess autonomic regulation of cardiovascular function ${ }^{2-4}$.

In this method, band of frequency marked as low frequency (LF, frequency range $0.04-0.15 \mathrm{~Hz}$ ) and high frequency (HF, frequency range $0.15-0.4 \mathrm{~Hz}$ ) are the components of total power (frequency range $0.00-0.4 \mathrm{~Hz}$ ) in a spectrum of 2-5 minutes record ${ }^{2-4}$. Measurements of two major LF and HF power components $\left(\mathrm{ms}^{2}\right)$, also expressed in normalized units (n.u) represent the relative value of each power component. The vagal activity is a major contributor to the HF component. The LF component is a marker of sympathetic modulation, but some suggest, as a parameter that include both sympathetic and vagal influences. $\mathrm{LF} / \mathrm{HF}$ ratio is considered as a mirror of sympatho-vagal balance ${ }^{2-3}$.

Previous studies reported higher LF and LF/HF ratio and significantly lower HF value \& HF nu in smokers $^{5}$. Only few studies investigated the acute effect of smoking on sympathetic and parasympathetic activity by HRV analysis. They found significant decrease in HF indicating 
decreased vagal activity and increased LF and $\mathrm{LF} / \mathrm{HF}$ ratio reflecting higher sympathetic discharge and impaired sympatho-vagal balance within 5 minutes after smoking which is clearly evident over 5 to 10 minutes. They suggested that cigarette smoking immediately alters cardiovascular autonomic regulation, which may cause cardiovascular complications ${ }^{6-7}$.

An investigation on the circadian variation on the reversal of HRV changes due to acute effect of smoking in taxi drivers observed that LF/HF ratio increased significantly within 5 minutes after smoking \& then returned to baseline within 15 minutes only at night. In daytime, HF increased but it remarkably decreased at night. These data suggest that smoking at night has a more potent acute effect on the cardiac modulation of taxi drivers than during the day ${ }^{8}$.

Very few studies reported acute effect with different duration of smoking. Some study involved very few sample with mixed gender and some study was done on taxi driver only. Therefore, this study has been designed to observe immediate impact of cigarette smoking on power spectral measures of HRV and the duration of the effect in male healthy current regular smokers.

\section{Methods}

This experimental study was carried out in the department of Physiology of Bangabandhu Sheikh Mujib Medical University (BSMMU) on apparently healthy 190 male subjects with age 20-55 years. Ethical issues of this study was approved by institutional review board of BSMMU. All subjects were current regular cigarette smokers \& they were hospital staffs of BSMMU, motor vehicle drivers and subjects living in Dhaka city with similar socio economic status. Informed written consents were taken from them. All subjects were free from history of active respiratory infection, coronary artery disease, congestive heart failure, Diabetes Mellitus, history of consumption of other tobacco products or alcohol, thyroid disorder, renal or hepatic dysfunction, history of taking drugs affecting CNS and psychiatric illness.

Data were recorded at baseline (before smoking) and successively $5 \mathrm{~min}$ and $30 \mathrm{~min}$ after completing a stick of cigarette.
The subjects were advised to follow several instructions from the previous night before the examination day. They were advised to have finished their meal by 9:00 pm and to have a sound sleep and should be refrain of any physical or mental stress and not to take any sedatives or any drugs affecting central nervous system and also advised to refrain from smoking cigarette for 12 hours before the nerve function tests done. Then he was asked to report at the Department of Physiology of BSMMU between 9:00 a.m. to11:00 a.m. on the day of examination. They were advised to take light breakfast in the morning without any tea, coffee or smoking on the test day. All the examination was done between 9 am to $2 \mathrm{pm}$ in the Autonomic Nerve Function Test laboratory under controlled laboratory condition.

After allowing to rest for 5 minutes blood pressure and pulse rate were recorded. Then the subject was prepared for recording HRV data. The subject was kept in supine position in a bed for 15-20 minutes in a cool, calm and controlled environment in the lab. During this period subject was advised to refrain from talking, eating or drinking and physical or any mental activity even sleep.

Then all preparations for recording of HRV parameters by a multichannel data acquisition device polyrite (RMS Polyrite) was finalized according to task force ${ }^{4}$. The values of power spectral HRV parameters such as LF (Low frequency), HF (high frequency), LF (nu), HF (nu), $\mathrm{LF} / \mathrm{HF}$ ratio was obtained by software analysis of RR intervals from 5 minutes recording of ECG. At first a baseline 5 min recording was taken keeping the subject in supine position. Then the subject was allowed to smoke one cigarette. HRV recording was taken 5 and 30 minutes after the cigarette was finished. Data were compared between before smoking and after 5 minute and then after 30 minutes successively.

Data were expressed as mean \pm SE. Comparison was performed by Paired t test.

$P$ value $<0.05$ was accepted as level of significance.

\section{Results}

In this study, significant elevation $(\mathrm{p}<0.001)$ of LF power, LF nu, LF/HF ratio and significantly decreased $(p<0.001)$ HF power, HF nu were 
Table I: Power spectral parameters of HRV before and after smoking at different periods $(\mathrm{n}=120)$

\begin{tabular}{lccc}
\hline Parameters & Pre-smoking & 5 min after smoking & 30 min after smoking \\
\hline LF power $\left(\mathrm{ms}^{2}\right)$ & $870.23 \pm 51.97$ & $1008.01 \pm 66.69^{* * *}$ & $871.13 \pm 56.4$ \\
HF power $\left(\mathrm{ms}^{2}\right)$ & $452.05 \pm 31.99$ & $390.05 \pm 29.91^{* * *}$ & $437.33 \pm 31.02$ \\
LF nu & $72.99 \pm 1.25$ & $77.56 \pm 1.16^{* * *}$ & $74.16 \pm 1.17$ \\
HF nu & $31.28 \pm 1.74$ & $27.2 \pm 1.84^{* * *}$ & $29.65 \pm 1.47$ \\
LF/HF ratio & $2.13 \pm 0.12$ & $2.85 \pm 0.15^{* * *}$ & $2.16 \pm 0.11$ \\
\hline
\end{tabular}

Data were shown as mean \pm SE. Statistical analysis was done by paired sample $t$ test

*5min after smoking vs pre-smoking $\left({ }^{* * *}=\mathrm{p}<0.001\right)$

observed in smokers after 5 min of smoking and then these values returned almost towards its pre smoking status after $30 \mathrm{~min}$ of smoking. (Table I).

\section{Discussion}

This present study investigated the immediate effect of cigarette smoking on cardiac autonomic nerve function in apparently healthy smokers by assessing power spectral measures of HRV after 5 minutes of complete smoking of a cigarette and then observed the recovery of acute effect by recording same data after 30 minutes of cessation of smoking. The present results of increased LF, LF nu, LF/HF and decreased HF, HF nu suggest significantly increased sympathetic and decreased parasympathetic activity immediately after smoking and the reversal of this changes towards its pre smoking value 30 minutes after cessation of smoking. These findings are parallel to the observation of some groups of authors ${ }^{1,6-}$ 7 . They also found increased sympathetic activity and decreased parasympathetic activity. Hayano $\&$ his groups also noted these autonomic changes 3 minutes after smoking ${ }^{1}$. Some author noticed autonomic changes begin during smoking and reaches its peak 5 to 10 minutes after smoking and returned to the baseline after 25 minutes. All these literatures viewed that all these changes are consequence of suppression of cardiac vagal activity after smoking which supports our results ${ }^{6-7}$.
The data after 5 mintues in this study clearly indicate immediate increase of sympathetic and fall of cardiac vagal activity and altered autonomic balance, All results after 30 mints demonstrated almost complete reversal of the cardiac autonomic change due to cigarette smoking.

Evidence from previous studies suggested that one of the major modifiable risk factors for cardiovascular disease is cigarette smoking ${ }^{5,9-}$ 10. It has a harmful effect on various parts of neuro-cardiovascular regulation system including afferent and efferent division of autonomic nervous system and central nervous system ${ }^{9}$.

Several researchers viewed that short term changes observed in LF/HF \& HF immediately after smoking are due to increased release and/ or a reduced clearance of catecholamine at neuroeffector junctions ${ }^{9,11}$. Moreover, nicotine dependent stimulation of sympathetic control and inactivation of vagal cardiovascular control is responsible for hemodynamic changes in smokers 9,12 .

Some other studies reported that nicotine is the main constituent of the cigarette for the cardiac sympathetic excitatory effects through increased release of catecholamines 9.14 .15 . This cardiac sympathoexcitatory effect of nicotine is the major reason for various hazardous effects of smoking $5,9,13$. This enhanced sympathetic activity increase heart rate and myocardial contractility

J Bangladesh Soc Physiol. 2018, June; 13(1): 8-12 
by acting on $\hat{a}_{1}$ adrenergic receptor and increase coronary vasomotor tone by acting on á 2 adrenoceptor $^{14-16}$.

Though the exact mechanisms entailed in the genesis of increased sympathetic and withdrawl of vagal activity during the period of smoking is not clearly evident, the contribution of nicotine for this deranged cardiovascular neural control has been most commonly highlighted. Research evidence suggested that nicotine stimulates the nicotinic receptors of autonomic ganglia as well as adrenal medulla resulting in increased cardiac sympathetic activity and increased release of catecholamine $9,15-16$, which supports the results of the current study. Nicotine may further intensify its action on peripheral chemoreceptors causing impaired baro-reflex sensitivity and attenuated vagal activity which is responsible for hemodynamic changes in smokers ${ }^{5,9}$ which may also be applicable for the smokers in the current study. In addition, the increased release of neuropeptide $\mathrm{Y}$ for autonomic balance causing suppression of cardiac vagal tone also contribute to the reduced vagal modulation in smokers ${ }^{17}$. The effect of monoamino oxidase inhibitors (MAO) like substances present in the tobacco smoke causes impairment of the cardiac autonomic nerve function by prolonging the effect of circulatory catecholamine ${ }^{18}$.

\section{Conclusion}

Increased sympathetic activity with concomitant suppression of cardiac vagal modulation occurs immediately after smoking a cigarette. Smoking on short term basis may cause an acute and transient decrease in cardiac vagal activity and also exaggerate cardiac sympathetic activity. The acute effect of smoking is reversed at about 30 minutes after smoking. Sympathovagal balance may shift towards the strong sympathetic dominance as a result of cigarette smoking. Therefore, chronic Cigarette smokers are more vulnerable to the risk of increased cardiovascular adverse event.

J Bangladesh Soc Physiol. 2018, June; 13(1): 8-12
Conflict of interest-The authors of this study have no conflict of interest.

\section{Author affiliations}

*1. Mehboba Ferdous, Assistant professor, Dept.of Physiology, Nightingale medical college, Ashulia, Dhaka.Email:mfurmi@yahoo.com

2. Sultana Ferdousi, Professor, Dept of Physiology, Bangabandhu Sheikh Mujib Medical, University, Dhaka, Bangladesh. Email:sferdousiratna@gmail. com; sferdousiratna@bsmmu.edu.bd

* For correspondence

\section{References}

1. Hayano J, Yamada M, Sakakibara Y, Fujinami T, Yokoyama K, Watanabe Y, Takata K. Short and long term effects of cigarette smoking on heart rate variability. The Am J Cardiol 1990; 65(1):84-8

2. Pomeranz B, Macaulay RJ, Caudill MA, Kutz I, Adam D, Gordon D, Kilborn KM, Barger AC, Shanon DC, Cohen RJ. Assessment of autonomic function in humans by heart rate spectral analysis. Am J Physiol Heart Circ Physiol 1985; 248: H151H 3

3. Akselrod S, Gordon D, Ubel FA, Shanon DC, Barger AC, Cohen RJ. Power spectrum analysis of heart rate fluctuation: a quantitative probe of beat to beat cardiovascular control.Science 1981;213:220-22

4. Task Force of the European society of cardiology and the North American society of pacing and electrophysiology. Heart Rate Variability. Standards of measurement, physiological interpretation and clinical use. Circulation 1996;93: 1043-65.

5. Cagirci G, CAY S, Karakurt O, Eryasar N, Kaya V, Canga A, Yesilay AB, Kilic H, Topaloglu S, Aras D, Demir AD, Akdemir R. Influence of heavy cigarette smoking on heart rate variability and heart rate turbulence parameters. Ann Noninvasive Electro cardiol 2009; 14(4):327-32

6. Karakaya O, Barutcu I, Kaya D, Esen AM, Saglam M, Melek M, Onrat E, Turkmen M, Esen OB, Kaymaz C. Acute effect of cigarette smoking on heart rate variability. Angiology 2007; 58: 620-4

7. Andrikopoulos GK, Dilaveris PE, Richter DJ, Gialafos EJ, Lazaki EA, Avgeropoulou CK. Influence of cigarette smoking on heart rate variability in young healthy subjects. ANE 1999;4(2).204-11

8. Kobayashi F, Watanabe T, Akamatsu Y, Furui H, Tomita T, Ohashi R, Hayano J. Acute effects of cigarette smoking on heart rate variability of taxi 
drivers during work. Scan J work Environ Health 2005;31(5):360-6

9. Alyan O, Kacmaz F, Ozdemir O, Maden O, Topaloglu S, Ozbakir C, Metin F, Karadede A, Iikay E. Effects of cigarette smoking on heart rate variability and plasma $\mathrm{N}$-terminal pro-B type natriuretic peptide in healthy subjects: is there the relationship between both markers? Ann Noninvasive Electrocardiol 2008; 13(2): 137-44

10. Barutcu I, Esen AM, Kaya D, Turkmen M, Karakaya O, Melek M, Esen OB, Basaran Y. Cigarette Smoking and Heart Rate Variability: Dynamic influence of Parasympathetic and Sympathetic Manuvers. ANE 2005; 10(3): 324-9

11. Grassi G, Seravalle G, Calhoun DA, Bolla GB, Giannattaiso C, Marabini M, Bo AD, Mancia G. Mechanisms responsible for sympathetic activation by cigarette smoking in humans. Circulation 1994;90:248-53

12. Benowitz NL. The role of nicotine in smokingrelated cardiovascular disease. Prev Med 1997;26(4):412-7
13. Hering D, Somers VK, Kara T, Kucharska W, Jurak P, Bieniaszewski L, Narkiewicz K. Symapthetic neural responses to smoking are age dependent. J hypertens2006; 24:691-5

14. Springer link Tobacco smoking and nicotine .Current concepts of the effects of nicotine on neurotransmitter release in the central nervous system .Advances in behavioral biology 1987; 31: 191-208

15. Adamopoulas D, Borne PV, Argacha JF.New insights into the sympathetic endothelial and coronary effects of nicotine. Clin exp pharmacol physiol 2008; 35:458-63

16. Katzung GB. Basic and Clinical Pharmacology $.11^{\text {th }}$ ed. New York: The McGraw-Hill Companies;2009. 94-108pp.

17. Ganong WF. Review of Medical Physiology. 23rd ed. USA: The Mc Graw-Hill Company; 2012. 556$8 \mathrm{pp}$.

18. News Medical. Nicotine Pharmacology [Internet] 2013[cited2011] Available from:http://www.new smedical.net/health/Nicotine-Pharmacology.aspx. 